\title{
Multichannel Spectral Image Enhancement for Visualizing Diabetic Retinopathy Lesions
}

Pauli Fält ${ }^{1}$, Masahiro Yamaguchi ${ }^{2}$, Yuri Murakami ${ }^{2}$, Lauri Laaksonen ${ }^{3}$, Lasse Lensu $^{3}$, Ela Claridge ${ }^{4}$, Markku Hauta-Kasari ${ }^{1}$, and Hannu Uusitalo ${ }^{5}$

1 University of Eastern Finland, School of Computing,

Yliopistokatu 2, P.O. Box 111, 80101 Joensuu, Finland

\{pauli.falt, markku.hauta-kasari\}@uef.fi

2 Tokyo Institute of Technology, Global Scientific Information and Computing Center 4259-S1-17 Nagatsuta, Midori-ku, Yokohama 226-8503, Japan

\{yamaguchi.m.aa,murakami.y.ac\}@m.titech.ac.jp

3 Lappeenranta University of Technology, Machine Vision and Pattern Recognition Laboratory, Skinnarilankatu 34, P.O. Box 20, 53851 Lappeenranta, Finland

$\{$ lauri.laaksonen, lasse.lensu\}@lut.fi

4 University of Birmingham, School of Computer Science, Edgbaston, Birmingham, B15 2TT, UK

e.claridge@cs.bham.ac.uk

5 Department of Ophthalmology, SILK, University of Tampere, School of Medicine,

Biokatu 14, 33014 University of Tampere, Tampere, Finland

hannu.uusitalo@uta.fi

\begin{abstract}
Spectral imaging is a useful tool in many fields of scientific research and industry. Spectral images contain both spatial and spectral information of the scene. Spectral information can be used for effective visualization of the features-of-interest. One approach is to use spectral image enhancement techniques to improve the diagnostic accuracy of medical image technologies like retinal imaging. In this paper, two multichannel spectral image enhancement methods and a technique to further improve the visualization are presented. The methods are tested on four multispectral retinal images which contain diabetic retinopathy lesions. Both of the methods improved the detectability and quantitative contrast of the diabetic lesions when compared to standard color images and are potentially valuable for clinicians and automated image analyses.
\end{abstract}

Keywords: spectral image, multispectral imaging, principal component analysis, enhancement, retina, diabetes mellitus, diabetic retinopathy.

\section{Introduction}

Spectral imaging is a powerful imaging modality, which allows one to capture both the spatial and spectral information of the target-of-interest. These data are often stored in a spectral image, i.e., a three-dimensional matrix where the first two dimensions (rows and columns) contain the spatial information of the target and the third dimension (layers) contains the information of the target's

A. Elmoataz et al. (Eds.): ICISP 2014, LNCS 8509, pp. 52-60, 2014.

(C) Springer International Publishing Switzerland 2014 
wavelength-dependent optical properties for each spatial location. Spectral imaging can be divided into multispectral or hyperspectral imaging depending on the number of unique spectral channels captured. Spectral imaging is used widely in many areas like in remote sensing, industrial quality inspection and medical imaging 123 .

Diabetes mellitus (DM) is one of the most important health care problems worldwide and diabetic retinopathy (DR) is the most common complication of DM 4 45. DR will reveal the overall status of DM and the vision threatening ophthalmological complications like proliferative DR and diabetic macular edema. Early detection of these complications is mandatory to avoid permanent loss of vision or expensive health care costs. Retinal imaging is a recommended tool for the screening of DR but has increasing potential to screen other eye or systemic diseases, e.g., age-related macular degeneration, glaucoma and systemic vascular and neurological diseases. Both greyscale and RGB images are used in clinical retinal imaging, even though the color information content of RGB images is very limited. Spectral images, however, typically contain tens or hundreds of individual color channels, providing detailed color information. Furthermore, spectral image enhancement can be applied to spectral images to enhance the contrast of the wavelength-dependent properties of the object in the images 6778910 . Enhanced spectral images of the retina could be used for early detection of DR.

In this study, two existing methods of single-channel spectral image enhancement were modified for multichannel spectral image enhancement [6] pared to the single-channel-enhancement methods, multichannel methods allow simultaneous enhancement of different spectral features. The two multichannel spectral image enhancement methods were applied to multispectral retinal images containing DR lesions in order to enhance the detectability of early-stage diabetic changes [11. Also, a scheme for further improving the results is introduced and applied to the multispectral retinal images.

\section{Multichannel Spectral Image Enhancement: Method 1}

The multichannel spectral image enhancement Method 1 is based on the approach introduced by Hashimoto et al. 6]. In their original method, a single spectral channel $i$ of the spectral image (a three-dimensional $X \times Y \times N$ data cube containing $X$ rows, $Y$ columns and $N$ spectral channels) is enhanced as follows: first, principal component analysis (PCA) is applied to the original spectral image and a spectral image estimate is calculated using $m$ principal components $(m<N)$ [12. This PCA reconstruction is also an $X \times Y \times N$ data cube. The PCA estimate is subtracted from the original spectral image:

$$
\mathbf{s}_{\mathrm{diff}}(x, y)=\mathbf{s}_{0}(x, y)-\hat{\mathbf{s}}(x, y),
$$

where $\mathbf{s}_{0}(x, y)$ is the spectrum from spatial coordinates $(x, y), x=1,2, \ldots, X$, $y=1,2, \ldots, Y$, from the original spectral image. Similarly, $\hat{\mathbf{s}}(x, y)$ is the spectrum from the estimated spectral image for the same coordinates $(x, y)$. 
A weighting factor matrix $\mathbf{W}$ is an $N \times N$ matrix of zeros, except for the $i^{\text {th }}$ column which is defined as

$$
[\mathbf{W}]_{i}=k \mathbf{g} .
$$

Here, constant $k$ is the weighting factor, and vector

$$
\mathbf{g}=\mathbf{s}_{\text {target }}-\mathbf{s}_{\text {mean }}
$$

is the difference between a selected target spectrum $\mathbf{s}_{\text {target }}$ and the mean spectrum $\mathbf{s}_{\text {mean }}$ of the original spectral image. Target spectrum is the spectrum of the color to be used for the enhanced visualization of the spectral features. The enhanced spectrum $\mathbf{s}_{\mathrm{enh}}(x, y)$ is defined as

$$
\mathbf{s}_{\mathrm{enh}}(x, y)=\mathbf{W} \mathbf{s}_{\mathrm{diff}}(x, y)+\mathbf{s}_{0}(x, y) .
$$

When matrix $\mathbf{W}$ is defined as in Eq. (2), the spectral image enhancement is applied to a single spectral channel $i$, and Eq. (4) can be written as

$$
\mathbf{s}_{\mathrm{enh}}(x, y)=k s_{\mathrm{diff}}(x, y, i) \mathbf{g}+\mathbf{s}_{0}(x, y)
$$

In this paper, matrix $\mathbf{W}$ is expanded for multichannel spectral enhancement:

$$
\mathbf{W}=\left[k_{1} \mathbf{g}, k_{2} \mathbf{g}, \ldots, k_{N} \mathbf{g}\right]=\mathbf{g k}^{\mathrm{T}}
$$

where vector $\mathbf{k}=\left[k_{1}, k_{2}, \ldots, k_{N}\right]^{\mathrm{T}}$ contains the weighting factors for the $N$ spectral channels.

Inserting Eq. (6) into Eq. (4), the enhanced spectrum can be calculated as

$$
\mathbf{s}_{\mathrm{enh}}(x, y)=\mathbf{g k}^{\mathrm{T}} \mathbf{s}_{\operatorname{diff}}(x, y)+\mathbf{s}_{0}(x, y) .
$$

If only one element of vector $\mathbf{k}$ is non-zero, the enhancement method of Eq. (7) reduces to the single-channel-enhancement method of Eq. (5).

\section{Multichannel Spectral Image Enhancement: Method 2}

The second multichannel spectral image enhancement method is based on the method introduced by Mitsui et al., in which a single spectral channel $i$ is also enhanced by using Eq. (44) [7]. In [7], the weighting factor matrix $\mathbf{W}$ is a $N \times N$ diagonal matrix with a single non-zero value $k$ at the $i^{\text {th }}$ row/column. Equation (4) becomes

$$
\mathbf{s}_{\mathrm{enh}}(x, y)=\mathbf{s}_{\mathrm{diff}}^{\prime}(x, y)+\mathbf{s}_{0}(x, y),
$$

where vector $\mathbf{s}_{\text {diff }}^{\prime}(x, y)$ contains one non-zero value $k s_{\text {diff }}(x, y, i)$ on its $i^{\text {th }}$ element. 
In Method 2, Eq. (8) is expanded for multichannel enhancement:

$$
\mathbf{s}_{\mathrm{enh}}(x, y)=\operatorname{diag}(\mathbf{k}) \mathbf{s}_{\operatorname{diff}}(x, y)+\mathbf{s}_{0}(x, y),
$$

where $\operatorname{diag}(\mathbf{k})$ contains vector $\mathbf{k}=\left[k_{1}, k_{2}, \ldots, k_{N}\right]^{\mathrm{T}}$.

\section{Scheme for Further Improving Object Visibility in Enhanced Spectral Images}

In order to further improve the visibility of selected features, the following steps were taken (see Fig. 1): The original spectral image can suffer from uneven lighting distribution due to uneven illumination conditions during imaging. Therefore, brightness normalization is applied to the spectral image. An illumination map is obtained for each spectral channel separately by convolving the original spectral channel image with a Gaussian kernel. The original spectral channel images are divided by their respective illumination maps to attain an even illumination field while preserving the image contrast.

The brightness-normalized spectral image and its PCA estimate were then used for the multichannel spectral image enhancement. Either Method 1 or Method 2 was used. Two different manually selected weight vectors $\left(\mathbf{k}_{1}\right.$ and $\mathbf{k}_{2}$ ) were used so that $\mathbf{k}_{1}$ enhanced the visibility of the features-of-interest and $\mathbf{k}_{2}$ enhanced the image background. Then, CIE (Commission Internationale de l'Eclairage) XYZ tristimulus values were calculated for the two enhanced spectral images according to the standard equations [13]:

$$
\{X(x, y), Y(x, y), Z(x, y)\}=\gamma \int_{\lambda} S(\lambda) R_{\mathrm{enh}}(x, y, \lambda)\{\hat{x}(\lambda), \hat{y}(\lambda), \hat{z}(\lambda)\} \mathrm{d} \lambda
$$

where $\lambda$ is wavelength, $(x, y)$ are pixel coordinates, $S(\lambda)$ is the spectrum of the light source (in this paper: $\mathrm{CIE}_{65}$ daylight illuminant), $R_{\mathrm{enh}}(x, y, \lambda)$ is the



Fig. 1. Flowchart of multichannel spectral image enhancement 
enhanced spectral image, and $\hat{x}(\lambda), \hat{y}(\lambda), \hat{z}(\lambda)$ are the spectral sensitivities of the CIE standard colorimetric observer. Here, it is assumed that $R_{\mathrm{enh}}(x, y, \lambda)$ is an enhanced spectral reflectance image. Normalization factor $\gamma$ is defined as $\gamma=100 /\left(\int_{\lambda} S(\lambda) \hat{y}(\lambda) \mathrm{d} \lambda\right)$. The XYZ-values were converted into RGB color space using the standard transformation. Finally, a difference image $D$ was calculated from the two RGB images as described in Fig. 1,

\section{Enhancement of Multispectral Retinal Images}

Multichannel spectral image enhancement Methods 1 and 2 were applied to a set of four previously acquired multispectral (spectral reflectance) images of the human retina. Detailed information on the acquisition of these images can be found from Ref. 11. These multispectral retinal images contained lesions related to DR. The size of the multispectral images was $800 \times 800 \times 30$, and these 30 spectral channels were obtained from the wavelength range $400-700 \mathrm{~nm}$ by $\sim 10 \mathrm{~nm}$ steps. Based on information from a previous study [11, the spectral channels corresponding to the following wavelengths were selected for spectral enhancement for Method 1: $\Lambda=[492,500,540,550,580,600,620] \mathrm{nm}$. For vectors $\mathbf{k}_{1}$ and $\mathbf{k}_{2}$, the following values were experimentally chosen for the selected wavelengths: $\left[\mathbf{k}_{1}\right]_{\Lambda}=[3,3,-3,-3,-3,3,-3]$, and $\left[\mathbf{k}_{2}\right]_{\Lambda}=[-1,-1,1,1,1,-1,1]$. For all the other wavelengths, the $k$-values were zero. To get high image contrast, the target color was set to black: $\mathbf{s}_{\text {target }}=\mathbf{0}_{N}$, where $\mathbf{0}_{N}$ is an $N$-vector of zeros.

For Method 2, the selected wavelengths and values for $\mathbf{k}_{1}$ were as follows: $\Lambda_{1}=$ $[492,500,540,550,580,600,620] \mathrm{nm},\left[\mathbf{k}_{1}\right]_{\Lambda_{1}}=[-7,-7,-7,14,14,-14,-14]$. Analogously, for vector $\mathbf{k}_{2}: \Lambda_{2}=[632,640,650,656,671,676,690,694] \mathrm{nm}$, and $\left[\mathbf{k}_{2}\right]_{\Lambda_{2}}=[-10,-10,-10,-10,10,10,10,10]$. For brightness normalization, the symmetric Gaussian kernel's size and standard deviation were both set to 200 pixels. The PCA estimates for the brightness-normalized spectral images were calculated using all except the three most significant principal components.

\section{Results and Discussion}

The results for four different multispectral retinal images - using the abovementioned vectors $\mathbf{k}_{1}$ and $\mathbf{k}_{2}$ - are shown in Figs. 2 and 3 for Methods 1 and 2, respectively. The RGB images of the spectral images enhanced by Eq. (7) and $\mathbf{k}_{1}$ are shown in Figs. $2(\mathrm{c}),(\mathrm{h}),(\mathrm{m})$ and $(\mathrm{r})$. The relative contrast of all bloodrelated features in the retina (blood vessel tree emerging from the optic disk, microaneurysms, bleedings, intraretinal microvascular abnormalities (IRMA)) is considerably improved, while the retinal background remains relatively unchanged. The RGB images for $\mathbf{k}_{2}$, Figs. 2(d), (i), (n) and (s), on the other hand, emphasize the retinal background and not the abnormalities caused by diabetes. The difference images in Figs. 2(e), (j), (o) and (t) have a diminished background variation for improved visibility and contrast of the blood-related retinal features. Analogously for Method 2 (Fig. 3). 


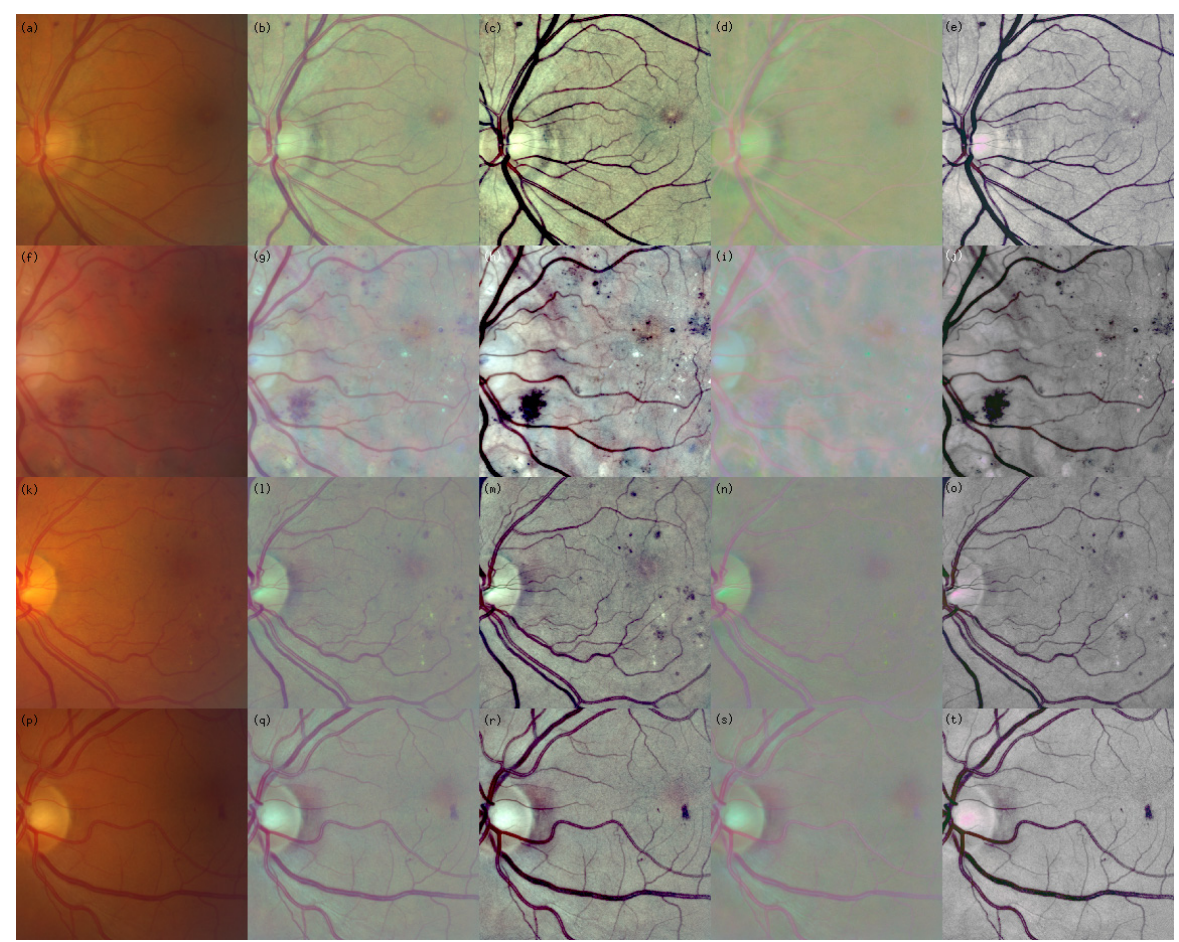

Fig. 2. Results for Method 1. Eye 1: (a) RGB image of the original multispectral image, (b) brightness-normalized image, (c) $\mathrm{RGB}_{1}$, (d) $\mathrm{RGB}_{2}$, and (e) difference image $D$. Eye 2: (f)-(j); Eye 3: (k)-(o); and Eye 4: (p)-(t).

For Eye 1, the multichannel spectral image enhancement reveals two microaneurysms in the macular area which are not visible in the original RGB image (Fig. 4). For Eye 2, the enhanced images show a large number of microaneurysms and small haemorrhages, and also a larger bleeding close to the optic disk. The choroidal background is relatively visible in the images (especially in Figs. 2(i) and $3(\mathrm{i})$ ). The relatively small, bright spots in the RGB images for Eye 2 and 3 are hard (lipid) exudates, which are another typical sign of DR. For Eye 3, the enhanced images again reveal a microaneurysms and small bleedings around the macula. And for Eye 4, microaneurysms and a larger bleeding in the macular area can be observed. Method 1 produces images with relatively high contrast between the blood-related features and the retinal background. The target color was black, which results in improved contrast. Method 2 doesn't use target color.

To quantify the improved contrast for the enhanced images, Michelson contrast was calculated on the luminance channel of the image in LAB-colorspace (Table 1). The color contrast was calculated as root-mean-square error (RMSE) contrast [14] with distance defined as CIEDE2000 color difference (Table 2). The decrease in contrast for Eye 1 is due to all the regions of interest where the contrast is calculated reside inside uniformly colored lesions. While the contrast with the background increases, the contrast inside the lesion area is lower. 


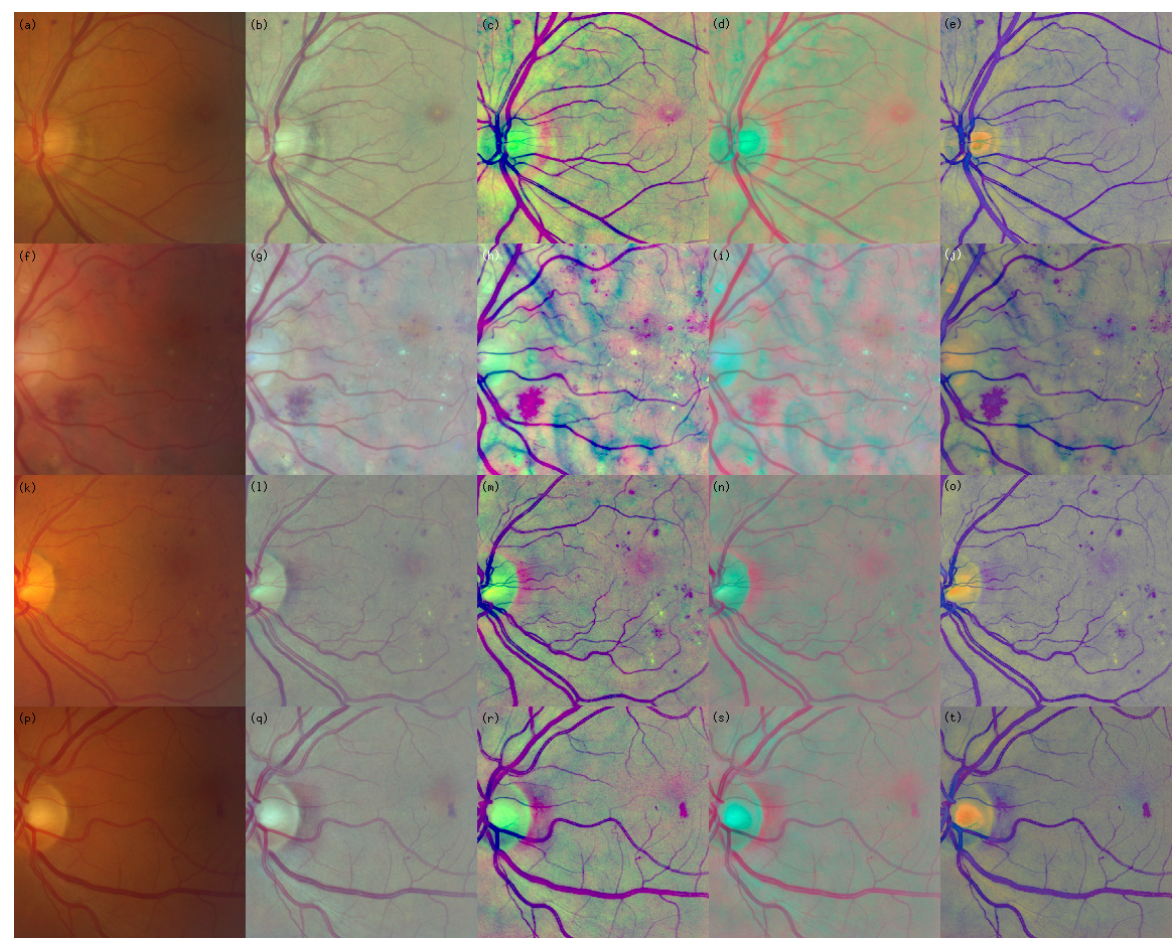

Fig. 3. Results for Method 2. Eye 1: (a) RGB image of the original multispectral image, (b) brightness-normalized image, (c) $\mathrm{RGB}_{1}$, (d) $\mathrm{RGB}_{2}$, and (e) difference image $D$. Eye 2: (f)-(j); Eye 3: (k)-(o); and Eye 4: (p)-(t).

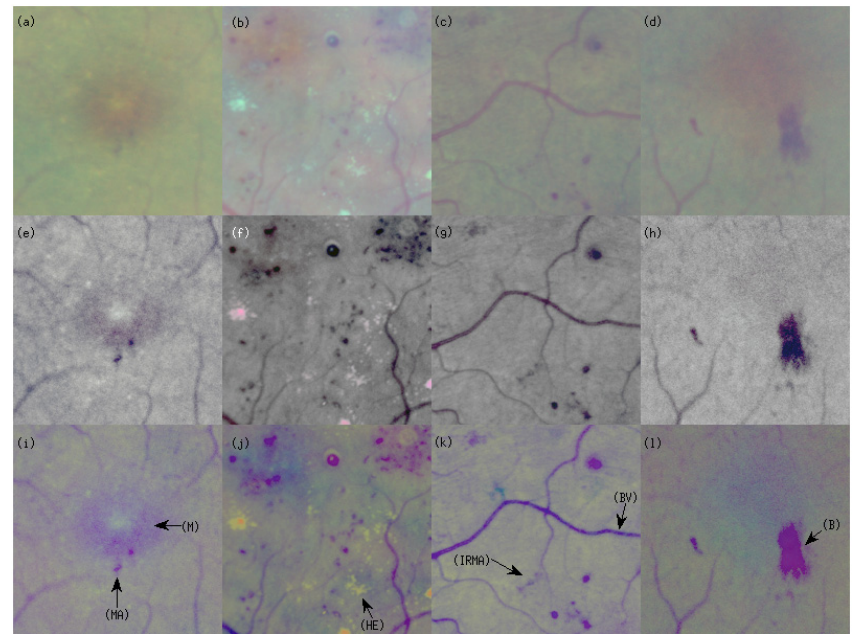

Fig. 4. Details for Eyes 1-4 from Figs. 2 and 3. (a)-(d): brightness-normalized images, (e)-(h) Method 1 results, and (i)-(l) Method 2 results. Arrows: macula (M), microaneurysm (MA), hard exudates (HE), IRMA, blood vessel (BV), and bleeding (B). 
Table 1. Michelson contrast (luminance). Means and standard deviations.

\begin{tabular}{c|cc|cc|cc}
\hline Eye & \multicolumn{2}{|c|}{ Brightness-norm. } & \multicolumn{2}{|c|}{ Method 1 } & \multicolumn{2}{c}{ Method 2 } \\
\hline & Mean & Std & Mean & Std & Mean & Std \\
1 & 19.57 & 1.89 & $\mathbf{2 6 . 3 7}$ & 5.20 & 15.36 & 12.08 \\
2 & 13.99 & 14.04 & $\mathbf{3 0 . 0 4}$ & 15.89 & 27.34 & 15.85 \\
3 & 13.94 & 5.88 & $\mathbf{3 5 . 8 8}$ & 13.16 & 31.63 & 9.82 \\
4 & 12.28 & 3.79 & 52.21 & 12.57 & $\mathbf{5 2 . 6 9}$ & 9.82 \\
\hline
\end{tabular}

Table 2. RMSE contrast (CIEDE2000). Means and standard deviations.

\begin{tabular}{c|cc|cc|cc}
\hline Eye & \multicolumn{2}{|c|}{ Brightness-norm. } & \multicolumn{2}{c|}{ Method 1 } & \multicolumn{2}{c}{ Method 2 } \\
\hline & Mean & Std & Mean & Std & Mean & Std \\
1 & $\mathbf{7 6 . 5 7}$ & 24.76 & 65.50 & 62.62 & 44.44 & 28.43 \\
2 & 31.29 & 15.58 & $\mathbf{4 1 . 8 3}$ & 31.44 & 30.20 & 15.18 \\
3 & 23.24 & 8.81 & 33.89 & 20.68 & $\mathbf{3 5 . 3 8}$ & 18.24 \\
4 & 34.57 & 19.65 & $\mathbf{5 7 . 4 1}$ & 35.97 & 46.14 & 8.59 \\
\hline
\end{tabular}

\section{Conclusion}

Two multichannel spectral image enhancement methods and a scheme for further improving the detectability of DR changes in retinal multispectral images were introduced. The introduced methods enhanced the detectability and contrast of blood-containing features in the retinal images, including typical signs of DR (microaneurysms, haemorrhages, retinal bleedings, IRMA). In some cases, lesions that were either poorly visible or not visible at all in the original image, became clearly visible in the enhanced images. The methods seem to have potential in clinical and automated retinal imaging as well as in other spectral images and spectral imaging applications. The future work will include quantitative validation of the methods by using a larger set of spectral retinal images.

Acknowledgments. The authors would like to thank the reviewers for their valuable comments and suggestions. This study was funded by the Academy of Finland (decision no. 259530).

\section{References}

1. Shaw, G.A., Burke, H.K.: Spectral imaging for remote sensing. Lincoln Laboratory Journal 14, 3-28 (2003)

2. Kukkonen, S., Kälviäinen, H., Parkkinen, J.: Color features for quality control in ceramic tile industry. Opt. Eng. 40, 170-177 (2001)

3. Li, Q., He, X., Wang, Y., Liu, H., Xu, D., Guo, F.: Review of spectral imaging technology in biomedical engineering: Achievements and challenges. J. Biomed. Opt. 18, 100901-1-100901-28 (2013)

4. Alberti, K.G.M.M., Zimmet, P.Z.: Definition, diagnosis and classification of diabetes mellitus and its complications. Part 1: Diagnosis and Classification of Diabetes Mellitus. Provisional Report of a WHO Consultation. Diabet. Med. 15, 539-553 (1998) 
5. Fong, D.S., Aiello, L., Gardner, T.W., King, G.L., Blankenship, G., Cavallerano, J.D., Ferris III, F.L., Klein, R.: Retinopathy in Diabetes. Diabetes Care 27, s84-s87 (2004)

6. Hashimoto, N., Murakami, Y., Bautista, P.A., Yamaguchi, M., Obi, T., Ohyama, N., Uto, K., Kosugi, Y.: Multispectral image enhancement for effective visualization. Opt. Express 19, 9315-9329 (2011)

7. Mitsui, M., Murakami, Y., Obi, T., Yamaguchi, M., Ohyama, N.: Color enhancement in multispectral image using the Karhunen-Loeve transform. Opt. Rev. 12, 69-75 (2005)

8. Nuffer, L.L., Medvick, P.A., Foote, H.P., Solinsky, J.C.: Multispectral/ hyperspectral image enhancement for biological cell analysis. Cytom. Part A 69A, 897-903 (2006)

9. Hollaus, F., Gau, M., Sablatnig, R.: Enhancement of multispectral images of degraded documents by employing spatial information. In: 12 th International Conference on Document Analysis and Recognition (ICDAR), pp. 145-149 (2013)

10. Yang, C., Lu, L., Lin, H., Guan, R., Shi, X., Liang, Y.: A fuzzy-statistics-based principal component analysis (FS-PCA) method for multispectral image enhancement and display. IEEE Trans. Geosci. Remote Sensing 46, 3937-3947 (2008)

11. Fält, P., Hiltunen, J., Hauta-Kasari, M., Sorri, I., Kalesnykiene, V., Pietilä, J., Uusitalo, H.: Spectral imaging of the human retina and computationally determined optimal illuminants for diabetic retinopathy lesion detection. J. Imaging Sci. Technol. 55, 030509-1-030509-10 (2011)

12. Tzeng, D.-Y., Berns, R.S.: A review of principal component analysis and its applications to color technology. Color Res. Appl. 30, 84-98 (2005)

13. Wyszecki, G., Stiles, W.S.: Color science: Concepts and methods, quantitative data and formulae. John Wiley \& Sons, Inc., New York (1982)

14. Peli, E.: Contrast in complex images. J. Opt. Soc. Amer. 7, 2032-2040 (1990) 